EOMmUn: Communication et organisation

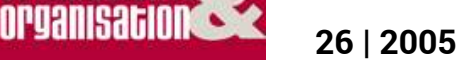

La communication des nouvelles éthiques de l'entreprise

\title{
La recherche et la pratique du management interculturel à la centrale nucléaire de Daya Bay
}

Lu Wei et Tang Yanzhao

(2) OpenEdition

Journals

Édition électronique

URL : http://journals.openedition.org/communicationorganisation/3288

DOI : 10.4000/communicationorganisation.3288

ISSN : 1775-3546

Éditeur

Presses universitaires de Bordeaux

Édition imprimée

Date de publication : 2 janvier 2005

Pagination : 189-200

ISSN : 1168-5549

Référence électronique

Lu Wei et Tang Yanzhao, « La recherche et la pratique du management interculturel à la centrale nucléaire de Daya Bay », Communication et organisation [En ligne], 26 | 2005, mis en ligne le 19 juin 2012, consulté le 19 avril 2019. URL : http://journals.openedition.org/

communicationorganisation/3288 ; DOI : 10.4000/communicationorganisation.3288

Ce document a été généré automatiquement le 19 avril 2019

(c) Presses universitaires de Bordeaux 


\title{
La recherche et la pratique du management interculturel à la centrale nucléaire de Daya Bay
}

\author{
Lu Wei et Tang Yanzhao
}

\section{NOTE DE L'ÉDITEUR}

Aide à la rédaction en français : Sophie Faure et Florence Hénon

\section{Introduction}

1 Nous nous proposons par cette recherche de formaliser une nouvelle méthode de management interculturelle qui pourrait être une référence lors de la construction et pour le management de futures centrales nucléaires en Chine. En nous référant à la théorie traditionnelle du management interculturel qui étudie la différence culturelle des nations, nous tenterons de construire un modèle structurant à partir de l'explicitation de la pratique du management interculturel à la centrale de Daya Bay.

2 La collecte des données a été effectuée à partir d'un questionnaire envoyé à 200 Chinois couvrant un large éventail de fonctions et de niveaux ainsi qu'à 50 Français qui ont travaillé en Chine. Cette démarche a été complétée par des interviews de 15 Chinois et 14 Français ayant occupé des fonctions élevées à des moments critiques dans le temps. Enfin, l'un des co-auteurs de l'article a participé lui-même au projet.

3 Nous nous proposons par cette recherche de formaliser une nouvelle méthode de management interculturelle qui pourrait être une référence pour la construction et le management de futures centrales nucléaires en Chine. 


\section{L'établissement du modèle}

4 Pour mieux faire comprendre notre théorie, nous la présentons ci-dessous sous forme d'un modèle structurant. Les principales composantes de CCIOT (Culture, Trust, Coopération, Objective, Intégration - Innovation) sont l'environnement culturel, la confiance, la coopération, l'objectif commun et l'intégration-innovation qui sont toutes subdivisibles. Le modèle CCIOT est divisé structurellement en trois niveaux: triangle stable, intégration-innovation et culture.

5 Le premier niveau, qui constitue le niveau de base du management interculturel - soit la stabilité - est composé des trois éléments :

- confiance

- coopération

- objectif commun

6 Pour atteindre l'objectif primordial et clairement défini des entreprises mixtes qui est de coopérer et de travailler conjointement, on ne peut dissocier ces trois éléments. La coopération et la confiance se déterminent par rapport à l'objectif commun dont la réalisation elle-même ne peut être garantie que par l'existence d'une réelle coopération et confiance. La coopération, la confiance et l'objectif commun constituent le triangle stable, base du management.

7 Le deuxième niveau est constitué par l'intégration-innovation et le triangle stable qui forment une composition «diamant». Les entreprises à capitaux mixtes réalisent l'intégration-innovation de ses environnements et de son personnel pluriels. Celles-ci profitent de ses ressources internes et externes; elles établissent de façon originale et durable l'organisation, la structure du management, le processus managérial, le système des ressources humaines et la culture d'entreprise.

8 L'intégration-innovation a un sens double :

- L'intégration, par la coopération et la compréhension des deux parties, se satisfait de l'une et l'autre.

- L'innovation, sur la base de l'intégration : les deux parties créent par des efforts communs un modèle particulier du management interculturel. Toutes les entreprises mixtes possèdent des facteurs innovateurs, puisqu'il s'agit d'une organisation nouvelle qui produit de nouveaux concepts, la réorganisation des ressources et de nouveaux règlements. Pour un développement de longue durée, ces entreprises cherchent des idées nouvelles, des produits nouveaux, des innovations.

9 L'intégration-innovation est indispensable au développement des entreprises interculturelles, quant au triangle stable, il l'est pour leur pérennité, par cette stabilité même qu'il leur procure. Le triangle stable est la base de l'intégration -innovation. Ces deux facteurs ne survivent pas isolément. L'intégration-innovation et le triangle stable constituent donc une composition « diamant ».

Le troisième niveau traite des relations entre la composition "diamant» et l'environnement culturel. Pour les entreprises mixtes, la culture influence chaque aspect de leur management. Les personnes de culture différente perçoivent la notion de confiance différemment. 


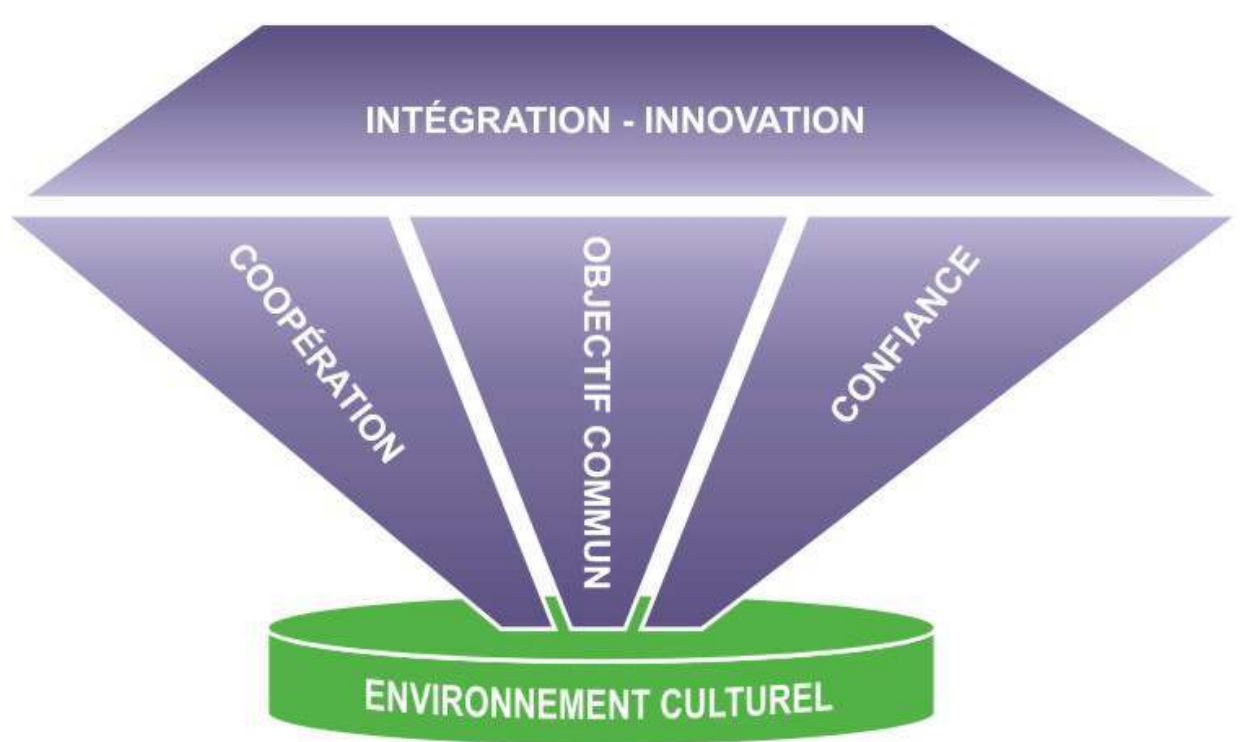

\section{Le modèle structurant du management interculturel}

11 En Chine, la confiance s'établit sur les relations du passé (la réputation personnelle et l'amitié d'autrefois) et les caractéristiques personnelles. Dans les pays occidentaux, la confiance est basée sur une institution. En Chine, les relations sont la base de la coopération. Pour réussir une coopération, il faut établir de bonnes relations.

En Occident, la coopération dépend du contrat. Pour commencer une coopération, il faut tout d'abord signer un contrat détaillé qui lie les deux parties. La culture influence l'environnement interne et la fonction managériale des entreprises interculturelles. Fons Trompenaars et Hampden-Turner soulignent dans leur livre que «dans toutes les cultures, on utilise pour la description du concept managérial les mêmes termes - tels que hiérarchie, autorité, créativité, management général de qualité, responsabilité - que les différentes cultures traitent différemment». Ce constat néglige les différentes bases culturelles sur lesquelles ces concepts s'établissent.

\section{L'objectif de coopération de longue durée établi conjointement par les Chinois et leurs partenaires étrangers}

Les partenaires des entreprises mixtes ont chacun leur propre motif et objectif. Percevoir ces entreprises comme ses propres enfants et parvenir à diminuer les différences culturelles entre elles, sont deux opérations de longue durée.

Les Chinois projetaient de construire une centrale nucléaire sur Daya Bay en automne 1978. Certains proposaient d'emprunter pour acheter des poulets dont la vente des oeufs permettrait le remboursement des emprunts contractés. Cette idée intéressait fort le directeur de CLP (China Light Power). M. Kadoorie, vieil homme âgé de plus de 80 ans, perspicace, avait constaté qu'à Hongkong, on produisait de l'électricité avec du pétrole dont le prix était en augmentation. Pour la diversification des sources d'énergie, il 
soutenait l'électricité nucléaire, mais comme il était impossible de trouver un site à Hongkong, il envisageait donc une coopération avec la province du Guangdong.

En ce qui concerne la coopération avec EDF, Framatome et GEC, il existait certes des conflits et des contradictions en raison des différences de culture et d'intérêts, mais la volonté de réussir était partagée. En effet, la sûreté nucléaire est un sujet commun au monde entier. La construction réussie d'une centrale nucléaire ne dépend pas d'un seul pays ou d'une seule entreprise. C'est une volonté mondiale. La construction d'une centrale nucléaire de qualité est l'objectif de tous les partenaires.

De plus, en France, le marché des centrales nucléaires est en saturation. L'industrie nucléaire française cherchait de nouveaux marchés à l'étranger. Comme la centrale de Daya Bay était le premier grand projet commercial de la France en Chine, le partenaire français voulait une réussite complète de ce dernier pour s'en servir comme référence pour de futurs projets. Enfin, chacun espère trouver le maximum de bénéfices dans l'opération. Il faut cependant prendre en considération les décisions de chacun et enfin réaliser l’objectif : « gagner ensemble».

\section{Les relations de coopération multilatérale}

17 Durant la construction de la centrale de Daya Bay, les coopérations sont multinationales et multilatérales. Outre la coopération avec China Power Plant, on peut citer entre autres :

- La coopération du gouvernement chinois avec les gouvernements français, anglais et hongkongais ; celle du gouvernement chinois avec le gouvernement du Guangdong et celle des ministères de l'industrie nucléaire, électronique et mécanique.

- La coopération de Guangdong Nuclear Power Joint Venture Co. Ltd avec EDF, FRA, ALSTOM et MITSUBISHI.

- La coopération avec de nombreuses entreprises nationales.

- Pour l'obtention de fonds, l'intervention de la Banque de Chine et de plusieurs banques internationales.

C'est une coopération d'hommes de talent venus du monde entier et de toute la Chine. L'esprit de coopération s'installe partout à Daya Bay.

\section{Les relations de confiance établies au cours de la coopération}

Durant la construction et l'exploitation de la centrale de Daya Bay, en raison des différences culturelles, des contradictions et des conflits se produisaient inévitablement. Certains Français se méfiaient de la compétence des Chinois et de même ces derniers ne comprenaient pas les manières de faire des expatriés. Au bout d'une période de contact, les malentendus se dissipèrent et la confiance s'installa. Ceci se manifeste par les volets suivants :

Premièrement, les Chinois et leurs partenaires étrangers avaient tous la volonté de construire une centrale de qualité et de sûreté optimales qui pouvait soutenir le développement régional et permettre une expérimentation pour de futures centrales nucléaires, par conséquent, ils travaillaient conjointement pour résoudre les problèmes. 
Deuxièmement, à Daya Bay, les Chinois et leurs partenaires étrangers prêtèrent tous de l'importance aux différences de culture et de valeurs. Une ambiance de discussion franche, d'écoute réciproque et de respect de la différence se créa dans le travail. Certains ingénieurs chinois nouèrent des liens d'amitié avec des expatriés.

A Daya Bay, on organise souvent des activités pour que les expatriés s'intègrent à la vie et la culture locale. Enfin, le cadre de travail et de vie est agréable. Le village des expatriés offre des conditions de vie correctes.

\section{L'analyse de la composition « diamant »}

Le triangle stable et l'intégration-innovation constituent une composition «diamant ». Ayant fait une analyse du triangle stable dans le texte ci-dessus, nous proposons maintenant une présentation de l'intégration-innovation qui contient celle du concept managérial, de l'organisation, de la gestion et de la culture.

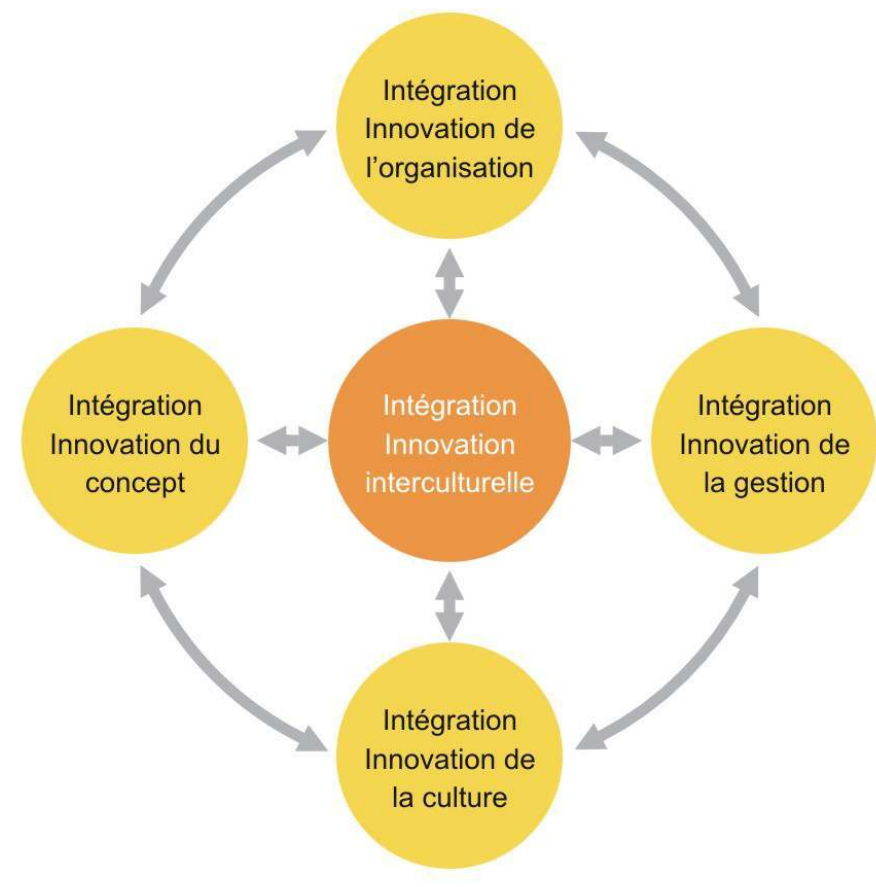

Les composantes de l'intégration-innovation

\section{L'intégration-innovation du concept managérial}

24 A la centrale de Daya Bay, malgré de grosses différences dans la perception du concept et de la méthode managériale, les Chinois et leurs partenaires étrangers ont la même volonté de coopération et d'objectif commun. Les agents chinois qui se situaient dans une période de transition (de l'économie planifiée à l'économie de marché) étudiaient consciencieusement les idées managériales étrangères et en tiraient profit. En parallèle, les expatriés essayaient d'appliquer leurs méthodes managériales en intégrant les conditions réelles de la Chine. Par la communication et la compréhension réciproques, ils sont parvenus à une identité des points de vue. 
En combinant les méthodes managériales de l'étranger avec la réalité chinoise et par l'intégration-innovation, la centrale de Daya Bay invente des idées managériales aux caractéristiques nucléaires.

\section{Maîtrise des risques et mesures préventives}

La centrale de Daya Bay est une entreprise de haute technologie conçue à partir d'emprunts de devises considérables, c'est pourquoi la notion de risque se manifeste non seulement dans la sûreté nucléaire mais aussi dans l'investissement et la gestion. Pour éviter et diminuer les risques, en toute circonstance, il faut faire un bilan des risques et en trouver la solution.

\section{Respect du contrat et des procédures}

27 C'est un engagement de la centrale qui a pour but de faire respecter les contrats, les règles et les normes. Tous les achats sont mis en adjudication. A la centrale de Daya Bay, tout s'effectue selon la procédure, c'est l'atout du management. La procédure managériale de Daya Bay se divise en trois niveaux (entreprise, centrale, site) et en trois catégories (management général, projet, contrat). Les niveaux et les catégories de même genre sont marqués par les mêmes numéros. La standardisation, la normalisation et la procédure garantissent le bon fonctionnement de la centrale.

\section{Contrôle interne}

$28 \mathrm{Au}$ commencement de la construction de la centrale, on a introduit à Daya Bay les systèmes managériaux de HKCLP et établi un strict contrôle interne qui couvre la sûreté nucléaire, l'assurance de qualité, la vérification des comptes, comptabilité et le contrôle de la discipline. A la centrale de Daya Bay, chaque département prend la responsabilité de son contrôle interne et reçoit le contrôle et la surveillance des autres départements. Ceci garantit le bon fonctionnement de la centrale et défend les intérêts de chaque partie.

\section{L'intégration-innovation de l'organisation}

La centrale de Daya Bay est une des premières entreprises mixtes de Chine. Son organisation a intégré et innové celle des pays développés. Les caractéristiques de son organisation se manifestent par l'application stricte du contrat et des règlements de joint-venture. Les pouvoirs des actionnaires, du conseil d'administration et du directeur général sont cadrés par la responsabilité, le pouvoir et l'intérêt de chacun. Les deux actionnaires de la compagnie joint-venture désignent chacun ses représentants pour le conseil d'administration. Entre le conseil d'administration et la direction, c'est purement une relation de mandatement. Le Conseil est une entité qui se charge de la surveillance, de la prise de décision et de la désignation de la direction. La direction gère l'entreprise en représentant le Conseil d'administration, les décisions hors de son pouvoir sont rapportées au conseil et celui-ci a le droit de récompenser ou de renvoyer les directeurs selon leur travail. 


\section{L'intégration-innovation de la gestion} de chaque culture qui fait surmonter ces barrières. A Daya Bay, l'intégration-innovation de la culture se réalise par les démarches suivantes :

- Faire pénétrer à Daya Bay des idées d'égalité culturelle qui signifient le respect des traditions culturelles (valeurs et mœurs culturelles) des partenaires. Le désir de calquer tout sur les pays occidentaux ou de persister dans une culture purement chinoise provoquerait de gros problèmes. C'est dans des relations basées sur l'égalité culturelle qu'on parvient à l'identité des points de vues.

- Assimiler les expériences avancées des pays étrangers : la Chine est un pays en développement. A Daya Bay, toutes les connaissances techniques et managériales ont été introduites des pays étrangers tels que la France et les Etats-Unis. Les agents chinois (du directeur aux ingénieurs) ont étudié consciencieusement ces connaissances.

- Les partenaires étrangers ont aussi beaucoup appris de nos pratiques. « Quiconque intervient dans le projet en Chine apprend quelque chose, quelquefois je trouve que les méthodes des Chinois sont aussi bonnes que les nôtres ", souligne un expert étranger.

\section{$2^{\text {ème }}$ démarche, la formation interculturelle}

La centrale de Daya Bay accorde de l'importance à la formation du personnel (de nouveaux agents et des agents en fonctionnement) et surtout à la formation du management interculturel au niveau de la maîtrise.

41 La langue est un outil de communication. La barrière de la langue constitue donc à Daya Bay un obstacle à la communication. Compte tenu de cette situation, la centrale a envoyé 
des ingénieurs apprendre le français ainsi que découvrir la culture française dans des instituts de langues. Sur le site et à Shenzhen, la centrale organisait régulièrement des formations aux langues française et anglaise dans le but de permettre à plus d'agents de communiquer directement avec les expatriés.

Sur le plan de la formation technique et managériale, la centrale faisait venir chaque année des professeurs des instituts spécialisés pour renouveler les connaissances et améliorer la compétence de ses agents. En 1998, la formation dans la centrale était en plein essor. 220 personnes suivaient à Shenzhen des cours dont les enseignants venaient de France, des Etats-Unis, du Canada, de Shanghai et de Pékin. La centrale a également envoyé les principaux acteurs techniques et managériaux dans les centrales nucléaires de France.

\section{$3^{\text {ème }}$ démarche, le respect de la différence culturelle}

Le respect de la différence culturelle est un facteur clé de la réussite de coopération. A Daya Bay, les Chinois et leurs partenaires étrangers prennent tous conscience de la différence de la culture et des valeurs. Dans le travail, on pratique la discussion franche, l'écoute réciproque, le respect de la différence culturelle et l'intégration des autres cultures.

\section{$4^{\text {ème }}$ démarche, la communication et la coordination interculturelles}

A Daya Bay, on organise souvent des activités qui permettent l'intégration des expatriés au cadre local et enrichissent la connaissance réciproque. Dans la prise de décision, la hiérarchie chinoise respecte les agents, leur contexte culturel et elle encourage la présentation des opinions diverses.

\section{$5^{\text {ème }}$ démarche, l'intégration interculturelle}

Les gens sont plus ou moins réticents vis-à-vis de la pénétration d'une autre culture. Daya Bay a pris les mesures suivantes:

- En organisant des rencontres et des activités diverses, la centrale a conduit ses agents à admettre les différences personnelles et culturelles. L'élimination des préjugés est la condition préalable à une coopération réussie.

- Le bon fonctionnement de la centrale est un sujet commun à tous. Mobiliser tout le monde pour la réalisation d'un objectif commun et faire d'un personnel d'entreprise où chacun possède sa propre personnalité, un groupe doté de valeurs et de convictions communes.

- La création d'un cadre de vie et de travail satisfaisant a également contribué à l'ensemble.

A la centrale de Daya Bay, dans chaque poste de travail, la responsabilité, les droits et la rémunération sont bien définis.

En dehors du travail, de nombreuses associations et animations enrichissent la vie des agents chinois et étrangers. Pour créer un cadre agréable pour les expatriés, la centrale a fait construire une base-vie qui possède un restaurant, une école et une clinique. Ceci facilite la vie des expatriés. 


\section{BIBLIOGRAPHIE}

Daniels, John D. and Radebaugh, Lee H., International Business, Environments and Operations, $8^{\text {th }}$ Edition, Addsion Weasley Longmann Inc., 1998

Hofstede, Gerd, Culture's Consequences : International Differences in Work-related Values, Beverly Hills, Calif :Sage, 1980

Ji Zuoru, Une perle brillante. La centrale nucléaire de Daya Bay, La Maison d'Edition de l'Orient, 1995, pp. 76-78 (en chinois)

$\mathrm{Pu}$ Jilong, Le recueil de Pu Jilong : Le retour d'expérience managerial à la centrale nucléaire du Guangdong , pp. 240-241 (en chinois)

Rong Jinben, Pu Wenchang, La revalorisation du capital : le modèle de Daya Bay, pp. 54-55 (en chinois)

Trompenaars, Fons, Turner Hampden, Riding the Waves of Culture. Understanding Cultural Diversity in Business, 2d Edition, London : Nicholas Brealey Publishing, 1998

Wrathall, Jeff and Berell, Michael M., International Management. Managing in the Era of Globalization, 2002

Xi Youmin, Han Wei, Etudes des Actions Managériales de l'Entreprise, Editions de l'Industrie Mécanique, 2004 (en chinois)

\section{RÉSUMÉS}

En nous référant à la théorie traditionnelle du management interculturel qui étudie la différence culturelle des nations, nous tenterons de construire un modèle structurant du management interculturel et d'analyser en recourant à ce modèle des actions du management interculturel sur le centrale de Daya Bay. Nous nous proposons par cette recherche de formaliser une nouvelle méthode de management interculturel qui pourrait être une référence pour la construction et le management des futures centrales nucléaires en Chine.

Referring to the traditional theory of the intercultural management that studies the cultural difference of the nations, we are tempted to establish a frame model of the intercultural management and resorting to this model analyzer the actions of intercultural management in Daya Bay Station. By this research, we intend to formalize a new model of intercultural management that may be a reference for the construction and management of the futures nuclear power plants in Chine.

\section{AUTEURS}

\section{LU WEI}

Lu Wei est Docteur de l'Université de Huazhong, Sciences et Technologies (HUST) et Directeur des services administratifs et adjoint à la Direction Générale de Daya Bay Nuclear Power Operations and Management Co., LTD. De 1989 à 1990, il a suivi une 
formation « EDF » en France. De 2003 à 2004, il a dirigé l'étude interculturelle sur l'expérience nucléaire franco-chinoise au sein de la centrale de Daya Bay. Mail : luwei@cgnpc.com.cn

\section{TANG YANZHAO}

Docteur en management et professeur adjoint à l'Université de Xiamen, il enseigne la théorie managériale, le management d'investissement et le management des entreprises internationales. Il est l'auteur d'articles sur le management dans plusieurs revues chinoises. 